\title{
Effects of Transition from Pediatrics to Adult Oriented Health Care of Patients with Chronic Disease: A Literature Review
}

\author{
Arathi Francis* \\ Western Sydney University, School of Nursing and Midwifery Teacher, Australia
}

*Corresponding author: Arathi Francis, Western Sydney University, School of Nursing and Midwifery Teacher, TAFE, NSW RN Prince of Wales Hospital, Australia.
Received Date: June 26, 2019

Published Date: July 08, 2019

\begin{abstract}
Pediatrics patients with chronic diseases have a higher prevalence of psychological, psychosocial and physical ill-health when compared with people in the general population. This significantly increases the rate of hospitalization and transition of patients with chronic disease from pediatric to adult-oriented health care. Transition of patients with chronic disease from pediatric to adult-oriented health care has remarkable effects on the patients involved and their families as well as the health care staff and health care system at large. This article explores the literature published between 2000 and 2015 in order to identify the effects of transition from pediatric to adult oriented health care of patients with chronic disease and discusses interventions needed for practice change to improve this transition. The effects of transition from pediatric to adult-oriented health care of patients with chronic disease are challenging to address. Existing literature does identify some interventions that can potentially provide the basis for practice change but fails to acknowledge the challenges faced by the nurses in the adult hospitals who are involved in the care of transition patients and their families. The result of this review revealed that it is crucial for health professionals to be aware of the challenges faced by the nurses in the adult hospitals who are involved in the care of transition patients and their families and implement appropriate interventions to improve this transition for both the patients and the nurses involved.
\end{abstract}

Keywords: Transition to Adult Hospitals; Effects of Transition; Patients with Chronic Diseases and Transition; Adult Nurses; Transition Patients

\section{Introduction}

\section{Aim}

The aim of this article is to review the existing literature in order to explore the effects of transition from pediatric to adultoriented health care of patients with chronic diseases. This article will also discuss the appropriate interventions identified in existing research that can improve the transition of patients with chronic diseases from pediatric to adult hospitals. This discussion aims to create awareness among the health care professionals especially the nurses, educators and academics to formulate effective educational programs to inform practice change and improve the transition from pediatric to adult oriented health care [1].

\section{Search strategy}

Initially, relevant texts were identified by general internet searches using Google Scholar. Then a systematic keyword search on CINAHL, Medline, Cochrane database, Nursing Ovid and PubMed was conducted in August 2015 to access literature relevant to the chosen topic. Keywords and Boolean operators such as "transition to adult hospitals" or "transition of pediatric patients" and "effects of transition" or "patients with chronic diseases and transition" were used to identify relevant literature. The search was restricted to full text publications between 2000 and 2015 and those published only in English. Abstracts of relevant articles were read in order to define appropriateness for inclusion in this paper. The identified articles were read thoroughly and critically analyzed for an effective qualitative study on the chosen topic.

\section{Results}

Transition of pediatric patients is not a one-off procedure but is a systematic gradual process [2]. This process should be well planned and timely to ensure effective roll over of care and 
ultimately achieving optimal health for patients with chronic disease [3]. Transition of patients with chronic disease from pediatric to adult-oriented health care has tremendous effects on the physical, psychological and psychosocial health of the involved patients and their families [4,5]. The financial impact of this transition of patients with chronic disease from pediatric to adultoriented health care on the health care system is of significance as it affects the economy of the country at large [6]. The outstanding result of this literature review is that the challenges faced by nurses in the adult hospitals, who are involved in the care of transition patients and their families, remains unexplored and needs attention to ensure optimal and holistic care for the transition patients and their families.

\section{Effects of transition on the involved patients and their families}

Transition, according to Blum et al. is defined as a multidisciplinary dynamic process wherein the therapeutic, psychosocial and scholastic needs of patients with chronic disease are met as they transfer from pediatric to adult oriented health care. The survival rate of children and youth with chronic illnesses and disabilities has significantly increased with further advancement in medical science and technology [7]. Consequently, there is an increase in the number of transitions of patients with chronic disease from pediatric to adult-oriented health care [8]. The uniqueness of the skill mix of the multidisciplinary staff of both the pediatric and the adult health care team is so vast that they have an inadequate understanding of each other's services and management in the care of transition patients $[9,10]$. The pediatric as well as the adult-oriented health care system along with the involved patients and their families are consequently faced with challenges in the management and accomplishment of effective transition. A well organized collaboration of services between the pediatric hospital and adult hospital is essential to ensure a smooth and effective transition of patients with chronic disease from pediatric to adult-oriented health care.

The very thought of transition from pediatric to adult hospital is overwhelming to most patients and families involved. This is evident in reports by patients with chronic disease, who themselves have indicated that the transition of patients with chronic disease from pediatric to adult-oriented health care is a frightening experience [11]. It means moving out of one's comfort zone to a completely new environment where one is suddenly exposed to the adult world - feeling abandoned and lonely - but expected to be an adult patient taking on self-responsibility. Separation from the treating physician and other pediatric health care team, unrealistic and wrong perception of receiving lesser care in the adult hospitals, irrational fear of being exposed to more infections in the adult hospitals and the fear of loss of parental involvement in the care and treatment are some of the major effects of transition on the patients and their families $[12,13]$.

A study by Geerts et al., (2008) showed that transition from pediatric hospitals to adult hospitals has a more robust effect on the parents of patients with chronic conditions than the actual patients. It has been found, however, that a systematic progressive transition from family focused care to an individual needs based approach will result in an effective transition from pediatric to adult hospital of patients with chronic disease [14]. This is supported by Lindsay and Hoffman, who claim that commitment and team work by the pediatric and adult health care team, along with family and patient involvement, will result in a successful transition from pediatric to adult hospital of patients with chronic disease ensuring optimal health and good quality of life for the patients and their families.

\section{Effects of transition on the health care system (financial impact)}

Apart from the physical and mental stresses faced by the involved patients and their families, a poorly organized transition has enormous financial impact on the health care system of the country. This is supported by [15], who claim that there is a significant increase in the annual cost to the health care providers and the health care system at large due to medical hospital admissions of transition patients with chronic disease. Poor and untimely transition increases the number of hospitalizations and readmissions to hospitals with complications, causing a negative effect on the health care system. This means the need for more resources to combat the complications, the need for more qualified and specialized healthcare professionals to care for the patients and families and more hospitalization time eventually adds further to the annual cost of the health care system. This is confirmed by the National Disability Services [16], New South Wales Health which is spending more than it should on the health and hospitalization of people with disability. The hospitalization and readmissions of such transition patients also affects other patients who are in more need of hospital-based care. Eventually there will be a significant impact on future health-care costs. This is reinforced by [17] who established that unplanned readmission rates and increased length of hospital stay of most patients with chronic disease conditions proportionally increases the financial burden on the health care system.

\section{Effects of transition on the nurses in the adult hospitals}

Knowledge deficit, insufficiency of skills required and lack of confidence in the management of transition patients with chronic disease and their families by nurses in the adult hospitals has not been addressed adequately. This has become a progressing inevitable challenge in providing optimal health to such transition patients and their families [18]. A face to face interview of nurses working in the neurological sciences ward of one of the tertiary teaching adult hospitals in Sydney, New South Wales- Australia, revealed that they feel unsupported and stressed both physically and mentally, in caring for the long-term transition patients and their families. These adult nurses also admitted that they were less empathetic in caring for long term transition patients and their families in comparison with other patients, because of the overwhelming and irrational demands and expectations of the transition patients and their families. The adult nurses also 
acknowledged that this lack of empathy reflected on the quality of care they provided to the long-term transition patients and their families. Therefore, it is evident that apart from the knowledge deficit, insufficiency of skills required and lack of confidence in the management of transition patients with chronic disease and their families, there are other challenges faced by the adult nurses such as emotional stress and difficulties in managing the involved families. These negative effects on the nurses in the adult hospitals remain unexplored. There is a lack of evidence regarding such physical and emotional stress experienced by the adult nurses in caring for transition patients and their families. Therefore, it is imperative that further research be conducted regarding the effects of transition on the nurses in the adult hospitals. However, this opinion is not limited to the adult nurses alone but extends to the other members of the adult health care team.

\section{Discussion}

Several articles describing the challenges faced by the transition of patients from the pediatric hospitals to adult hospitals highlight the fact that it is a complex process involving a multidisciplinary approach [19,20]. The literature reveals that a number of interventions can be implemented to bring about an effective transition of patients with chronic disease from pediatric to adultoriented health care. Most of the interventions implemented are to address and minimize the effects of transition on the involved patients and their families and the health care system. However, the effects of such transition patients and their families on the nurses in the adult hospitals are not well detailed. Some of the interventions implemented to address and minimize the effects of transition on the involved patients and their families and the health care system include programs such as formulation and implementation of transition protocols and readiness programs, health education and health promotion programs to involved patients and families and education and training of health care team especially the adult nurses.

\section{Formulation and implementation of transition protocols and readiness programs}

Formulation and implementation of transition protocols and readiness programs involve a clear understanding of the different phases in the transition process. According to Baines there are three main phases in the transition process namely the beginning phase, the middle phase and the end phase. The beginning phase is where the decision to make the transition is made, followed by the middle phase where the preparation of the involved patient and their families or care providers is carried out and the end phase where the involved patient is actually transferred to the adult care hospital for further management .

Transition protocols such as transition care clinical pathways and readiness programs such as the readiness to graduate questionnaires primarily assess the ability of the involved patients and their families to make appropriate health decisions in collaboration with the multidisciplinary health care professionals. These transition protocols paved the way to improving the quality and continuity of care of the transition patients and their families $[21,22]$. The transition protocols and readiness programs lead to successful and effective transitions and management of the involved patients and their families only when there is a professional collaboration between the multidisciplinary health team of both the pediatric as well as the adult hospitals. This is reinforced by [23] who highlighted that the dynamics of an interdisciplinary team approach is essential to a successful transition.

\section{Health education and health promotion programs to involved patients and families}

Health education and health promotion programs are essential to achieve successful transition of involved patients and their families to adult hospitals. [24] indicated that comprehensive education which encourages independence and self-responsibility of the involved patients is certainly an absolute necessity to emphasize the importance of ongoing care. This view is also supported by Shah, Parag, and [25] who emphasize that the education and promotion programs conducted for a successful transition should bring about an improvement in the involved patients' self-confidence, autonomy, and ability to self-advocate. Creating awareness and educating about the differences between the pediatric and the adult care management along with the other issues of adult life like higher studies, sexuality, family planning and employment should also be addressed in the patient education and health promotion programs [26]. Parental education and support make the transition easier and effective [27]. Education about the availability of other social services such as education, job development and training, rehabilitation, community-based parent groups and carer's support groups within the community should also be addressed. This is supported by who suggested that health care professionals should co-ordinate these services and make referrals as needed to ensure that the involved patients and their families will manage independent living and enjoy better quality of life.

\section{Education and training of health care team especially the adult nurses}

Knowledge deficit regarding the multifaceted medical and psychosocial needs of the transition patients and the care expectations from their families is one major challenge faced by the adult health care providers [28]. This advocates the importance of involving the adult health care providers especially the adult nurses in the transition process. Evidence suggests that the adult nurses need specialized education and training in meeting the specific needs of the transition patients and their families [29]. Although evidence-based research highlights the efficiency of education and training programs in making an effective transition from pediatric to adult hospitals, there is not much evidence about specialized training of adult nurses in the management of transition patients and their families. The absence of evidence-based training programs justifies the need for more specific educational and training programs for adult nurses targeted towards management and care of transition patients and their families. 


\section{Limitation of the Study}

This review is restricted to studies conducted within a limited time frame specifically between the year 2000 to 2015 and those written in English. Results from this review are limited to the effects of transition on the involved patients and their families, and the adult nurses alone. The effect of transition on the other health care team is not addressed in this review.

\section{Conclusion}

In summary, this literature review found that few studies have evaluated the effects of transition from pediatric to adult-oriented health care of patients with chronic disease and their families while others have valued the impact of effective interventions on the outcome of transition from pediatric to adult-centered health care. The major effects of transition on the involved patients and their families are fear of the unknown, separation from the treating team and un-readiness to take responsibilities in the adult world. These can be significantly resolved by professional collaboration of the pediatric as well as the adult health care providers and the active participation of the involved patients and their families in the formulation and implementation of effective transition protocols and readiness programs. Significant increase in the annual cost is the main financial impact of transition on the health care system. Timely and well organized transitions will help reduce this financial burden by significantly reducing the hospital stay and readmission of such patients.

The outstanding finding of this literature review is that the challenges especially the physical and mental stresses faced by nurses in the adult hospitals who are involved in the care transition patients and their families remain unexplored. This provides a basis for forthcoming research that can study the challenges faced by nurses in the adult hospitals who are involved in the care transition patients and their families and develop strategies to ensure effective transition of patients from pediatric hospitals to adult care hospitals. Thus making transition from pediatric to adultoriented health care of patients with chronic disease a pleasant and rewarding experience to all the people involved namely the patients, their families, and the health care team especially the adult nurses involved in the care of such transition patients and their families.

\section{Acknowledgements}

I thank my Unit Coordinator Judy Mannix for her continued guidance and timely support. I am grateful to my night duty colleagues in neurological sciences ward at Prince of Wales Hospital and my family for their support and encouragement.

\section{Conflict of interest}

The author reports no conflicts of interest. The author is solely responsible for the content and writing of this paper.

\section{References}

1. Mc Laren S, Belling R, Paul M, Ford T, Kramer T, Weaver Singh S P (2013) Talking a different language: An exploration of the influence of organizational cultures and working practices on transition from child to adult mental health services. BMC Health Serv Res 13(1): 254.
2. Lindsay S, Hoffman A (2015) A complex transition: Lessons learned as three young adults with complex care needs transition from an inpatient paediatric hospital to adult community residences. Child Care Health Dev 41(3): 397-407.

3. Stewart D (2009) Transition to adult services for young people with disabilities: Current evidence to guide future research. Dev Med Child Neurol 51(4): 169-173.

4. Brumfield K, Lansbury G (2004) Experiences of adolescents with cystic fibrosis during their transition from paediatric to adult health care: A qualitative study of young Australian adults. Disabil Rehabil 26(4): 223234.

5. Crowley R, Wolfe I, Lock K, McKee M (2011) Improving the transition between paediatric and adult healthcare: A systematic review. Arch Dis Child 96(6): 548-553.

6. Gardiner C, Brereton L, Frey R, Wilkinson-Meyers L, Gott M (2014) Exploring the financial impact of caring for family members receiving palliative and end-of-life care: A systematic review of the literature. Palliat Med 28(5): 375-390.

7. Khan A, Baheerathan A, Hussain N, Whitehouse W (2013) Transition of children with epilepsies to adult care. Acta Paediatr 102(3): 216-221.

8. Baines J M (2009) Promoting better care: Transition from child to adult services. Nurs Stand 23(19): 35-40.

9. Harden PN, Nadine P (2006) Pediatric to adult transition: A personal experience. Prog Transplant 16(4) 324-328.

10. Rutishauser C, Akré C, Surìs J (2011) Transition from pediatric to adult health care: Expectations of adolescents with chronic disorders and their parents. Eur J Pediatr 170(7): 865-871.

11. Minicozzi A (2000) Transition to adult care: Another view. Pediatric Nursing 26(4): 411.

12. Geerts E, Van De Wiel H, Tamminga R (2008) A pilot study on the effects of the transition of paediatric to adult health care in patients with haemophilia and in their parents: Patient and parent worries, parental illness-related distress and health-related quality of life. Haemophilia 14(5): 1007-1013.

13. Montalembert M, Guitton C, French Reference Centre for Sickle Cell Disease (2014) Transition from paediatric to adult care for patients with sickle cell disease. Br J Haematol 164(5): 630-635.

14. Rapley P, Davidson P (2010) Enough of the problem: A review of time for health care transition solutions for young adults with a chronic illness. J Clin Nurs 19(3-4): 313-323.

15. Gardner R, Li Q, Baier R R, Butterfield K, Coleman E A, Gravenstein S (2014) Is implementation of the care transitions intervention associated with cost avoidance after hospital discharge? J Gen Intern Med 29(6): 878-884.

16. National Disability Services (2014) People with disability and hospitalisation: Challenges and opportunities in NSW NDS Background Paper.

17. Wong E L, Cheung A W, Leung M C, Yam CH, Chan F W, et al. (2011) Unplanned readmission rates, length of hospital stay, mortality, and medical costs of ten common medical conditions: A retrospective analysis of Hong Kong hospital data. BMC Health Serv Res 11: 149.

18. Viner R (1999) Transition from paediatric to adult care. Bridging the gaps or passing the buck? Arch Dis Child 81(3): 271-275.

19. McDonagh J E (2005) Growing up and moving on: Transition from pediatric to adult care. Pediatr Transplant 9(3): 364-372.

20. Stabile L, Rosser L, Porterfield K M, McCauley S, Levenson C, et al. (2005) Transfer versus transition: Success in pediatric transplantation brings the welcome challenge of transition. Prog Transplant 15(4): 363-370.

21. Gravelle A M, Paone M, Davidson AGF, Chilvers MA (2015) Evaluation of a multidimensional cystic fibrosis transition program: A quality improvement initiative. J Pediatr Nurs 30(1): 236-243.

22. Huang J S, Tobin A, Tompane T (2012) Clinicians poorly assess health literacy-related readiness for transition to adult care in adolescents with 
inflammatory bowel disease. Clinical Gastroenterology and Hepatology $10(6)$ : 626-632.

23. Cerns S, McCracken C, Rich C (2013) Optimizing adolescent transition to adult care for sickle cell disease. Medsurg Nurs 22(4): 255-257.

24. Clarizia NA, Chahal N, Manlhiot C, Kilburn J, Redington A N, et al. (2009) Transition to adult health care for adolescents and young adults with congenital heart disease: Perspectives of the patient, parent and health care provider. Can J Cardiol 25(9): e317-e322.

25. Shah P, Boudos R (2012) Transitions from adolescent to adult care. Pediatr Ann 41(2): 73-78.

26. Jalkut M K, Allen P J (2009) Transition from pediatric to adult health care for adolescents with congenital heart disease: A review of the literature and clinical implications. Pediatr Nurs 35(6): 381-387.
27. Betz CL (2004) Adolescents in transition of adult care: Why the concern? Nurs Clin North Am 39(4): 681-713.

28. Gilliam PP, Ellen JM, Leonard L, Kinsman S, Jevitt C M, et al. (2011) Transition of adolescents with HIV to adult care: Characteristics and current practices of the adolescent trials network for HIV/AIDS interventions. J Assoc Nurses AIDS Care 22(4): 283-294.

29. O Sullivan Oliveira J, Fernandes S M, Borges L F, Fishman LN (2014) Transition of pediatric patients to adult care: An analysis of provider perceptions across discipline and role. Pediatr Nurs 40(3): 113-120. 